\title{
HAJED - TDAH: Heurísticas para Avaliação de Jogos Educacionais Digitais para Pessoas com TDAH
}

\author{
Laura F. Silva, Diego Barbosa B. da Costa, Ana Carolina G. Inocêncio \\ ${ }^{1}$ Curso de Bacharelado em Ciências da Computação - Universidade Federal de Goiás \\ (UFG)/Regional Jataí \\ Caixa Postal 03 - Jataí - GO - Brasil \\ \{laurafsilva1, diegocostacmp, anacarolina.inocencio\}@gmail.com
}

\begin{abstract}
The games created to Attention-deficit/hyperactivity disorder (ADHD) people have become a subject for research in areas such as Education, Neuroscience, Software Engineering, among others. In Education, these games are called Digital Educational Games (DEG). Similar to other types of software, these games also require a quality. However, there is a lack of appropriate tools to assist in the process of evaluation of DEG for ADHD people. The proposal for this question, presented by this study is to use the technique of software usability inspection, which can also be used to evaluate games, titled as Heuristic Evaluation.
\end{abstract}

Resumo. Os jogos criados para pessoas com Transtorno de Déficit de Atenção e Hiperatividade (TDAH) tem se tornado assunto para pesquisas em áreas como Educação, Neurociência, Engenharia de Software, dentre outras. $\mathrm{Na}$ Educação, esses jogos são denominados Jogos Educacionais Digitais (JEDs). E como os demais tipos de softwares, esses jogos também necessitam de uma avaliação de qualidade. No entanto, faltam ferramentas apropriadas para auxiliar no processo de avaliação de JEDs para pessoas com TDAH. A proposta para esta questão, apresentada por este trabalho foi utilizar a técnica de inspeção de usabilidade de softwares, mas que pode ser utilizada para avaliar jogos, intitulada como Avaliação Heurística.

\section{Introdução}

A área dos jogos vem traçando novas interfaces com outros campos do saber, sendo assim, alguns ambientes de jogos estão sendo produzidos para potencializar aspectos motores, sociais, culturais, afetivos e cognitivos (ROCHA; FILHO; ALVES, 2014). Como é o caso de jogos desenvolvidos para pessoas com Transtorno de Déficit de Atenção e Hiperatividade (TDAH) que se tornaram alvo para pesquisadores de diversas áreas como Educação, Psicologia, Neurociência, Engenharia de Software, Realidade Virtual, dentre outras. (ALVES; BONFIM, 2016; OLIVEIRA; ISHITANI; CARDOSO, 2013; RIVERO; QUERINO; STARLING-ALVES, 2012; GUIMARÃES; RIBEIRO, 2010).

Consequentemente, como ocorre nos demais tipos de softwares, os Jogos Educacionais Digitais (JEDs) voltados para pessoas com TDAH também necessitam de uma avaliação de qualidade, cujo assunto é o objeto de estudo deste trabalho. Essa avaliação ocorre durante o ciclo de desenvolvimento do software e tem como objetivo 
validar as funções e características especificadas na fase de levantamento dos requisitos. Porém, o problema relacionado a esse tema, diz respeito a falta de ferramentas apropriadas para auxiliar no processo de avaliação e desenvolvimento de jogos específicos para TDAH (ROCHA; BITTENCOURT; ISOTANI, 2015; OLIVEIRA; ISHITANI; CARDOSO, 2013; BASTOS et al., 2012).

Portanto, experiências e conclusões de estudos sobre como o transtorno afeta no aprendizado, podem levar os avaliadores e desenvolvedores desses jogos, à atender melhor as necessidades pedagógicas e de apoio terapêutico para esse público (OLIVEIRA; ISHITANI; CARDOSO, 2013).

Neste contexto, o objetivo geral deste trabalho é apresentar o conjunto de heurísticas HAJED-TDAH, destinado à avaliação e desenvolvimento de JEDs para pessoas com TDAH. Esse conjunto foi criado com o auxílio de especialistas em TDAH e também seguindo as consideração de Annetta (2010) sobre as características indispensáveis de um bom JED, que foram adaptadas atentando para as dificuldades das pessoas com TDAH, por meio dos trabalhos de Souza et al. (2010), Valle et al. (2013) e Alves e Bonfim (2016).

Para melhor explanar sobre o conjunto de heurísticas HAJED-TDAH, este artigo está dividido da seguinte forma: Na Seção 2 é apresentado o referencial teórico, com o intuito de expor a relevância do conjunto de heurísticas HAJED-TDAH; a Seção 3 apresenta o conjunto de Heurísticas HAJED-TDAH, a avaliação é apresentada na Seção 4 e as considerações finais e trabalhos futuros são apresentadas na Seção 5.

\section{Referencial Teórico}

O TDAH exerce um forte impacto nas atividades educacionais, ocasionando dificuldades no aprendizado e no relacionamento do estudante com os colegas e professores (SILVA; PAULA; SARLAS, 2011), o que é resultado de alterações específicas provocadas pelo transtorno nas Funções Executivas ${ }^{1}$ (FE). No entanto, estudos sobre as possíveis contribuições dos jogos para o desenvolvimento das FEs (ROCHA; FILHO; ALVES, 2014), propõem uma melhora significativa da atenção, dos processamentos visuais e espaciais e, principalmente, da memória operacional (RIVERO; QUERINO; STARLING-ALVES, 2012).

Nesse sentido, uma alternativa viável para auxiliar no ensino e aprendizagem de pessoas com TDAH seria utilizar os Jogos Educacionais Digitais (JEDs). Jogos, desse tipo, são considerados jogos sérios (Serious Games), ou seja, jogos que tem um propósito além do entretenimento. (ROCHA; BITTENCOURT; ISOTANI, 2015).

Os JEDs são uma ferramenta atrativa, dinâmica e motivadora que podem contribuir consideravelmente no processo de ensino e aprendizagem de pessoas com TDAH, por possuírem benefícios favoráveis para que se trabalhe as dificuldades causadas pelo transtorno. Mas, mesmo com tantos aspectos positivos, muitos jogos com o propósito sério, como os JEDs, não alcançam os objetivos para os quais foram

\footnotetext{
${ }^{1}$ Funções executivas: constituem um conjunto de processos cognitivos que permitem a regulação da cognição e do comportamento, possibilitando o engajamento do indivíduo em ações complexas.
} 
VI Congresso Brasileiro de Informática na Educação (CBIE 2017)

Anais do XXIII Workshop de Informática na Escola (WIE 2017)

projetados. (LEITE; MENDONÇA, 2013). Dentre as principais razões, pode-se destacar a simplicidade destes jogos, quando comparados aos jogos comerciais, o que pode desmotivar os usuários, ou também, por proporem tarefas repetitivas.

Para evitar situações como essas e garantir qualidade aos JEDs, Annetta (2010) disponibilizou uma lista com seis elementos indispensáveis a esses jogos, sendo eles: identidade; imersão; interatividade; maior complexidade; análise de desempenho; e instrutivo. Esses elementos podem ser utilizados, de maneira adaptada, para conduzir com segurança o desenvolvimento e a avaliação de JEDs para TDAH.

Uma forma eficiente de realizar a avaliação de JEDs para pessoas com TDAH, conforme proposto por este estudo, é de se utilizar a Avaliação Heurística, uma técnica de inspeção de usabilidade de softwares, criada por Nielsen (1994). A Avaliação Heurística, possui algumas vantagens em relação as outras técnicas, como ser simples, de menor custo, rápida e a possibilidade de ser utilizada por avaliadores especialista e não especialistas em qualquer fase do ciclo de desenvolvimento de um projeto (NIELSEN, 1994).

Sendo assim, o conjunto de heurísticas HAJED-TDAH tem como finalidade possibilitar a especialistas e não especialistas uma avaliação eficiente de JEDs voltados para pessoas com TDAH, podendo também ser utilizado como guia para desenvolvimento.

\section{Heurísticas HAJED-TDAH}

O conjunto de heurísticas HAJED-TDAH (Heurísticas para Avaliação de Jogos Educacionais Digitais para pessoas com TDAH), foi fundamentado com base em: i) experiências de especialistas em TDAH; ii) trabalhos sobre heurísticas para jogos digitais e também relacionados a JEDs para pessoas com TDAH; e iii) características que bons JEDs devem possuir (ANNETTA, 2010).

Assim, o conjunto de heurísticas HAJED-TDAH pode ser dividido em três categorias distintas, com base no processo de construção das mesmas, sendo essas classificadas em: combinadas a partir de conjuntos diferentes de heurísticas para avaliação e desenvolvimento de JEDs; criadas com base em estudos que tratam sobre jogos e TDAH ou de forma exclusiva por especialistas no tratamento de pessoas com TDAH; e adaptadas para pessoas com TDAH, a partir de heurísticas já existentes. A Tabela 1 apresenta o conjunto de heurísticas HAJED-TDAH.

Tabela 1. Conjunto de heurísticas HAJED-TDAH

\begin{tabular}{|l|c|}
\hline \multicolumn{1}{|c|}{ HEURÍSTICA COMBINADAS - HAJED-TDAH } & Referência \\
\hline $\begin{array}{l}\text { H01- O jogo apresenta elementos apontadores (setas, mapas, avisos, etc.) sobre } \\
\text { a localização e situação do jogador, que despertam a atenção e auxiliam o } \\
\text { mesmo. }\end{array}$ & $\begin{array}{l}\text { [Valle et. al, 2013] } \\
\text { [Souza et. al, 2010] }\end{array}$ \\
\hline $\begin{array}{l}\text { H02- O jogo oferece liberdade de decisões ao jogador sem que isso o prejudique } \\
\text { no cumprimento de tarefas necessárias, e sem deixá-lo confuso quanto à } \\
\text { compreensão do objetivo final. }\end{array}$ & $\begin{array}{l}\text { [Valle et. al, 2013] } \\
\text { [Souza et. al, 2010] }\end{array}$ \\
\hline $\begin{array}{l}\text { H03- Os elementos presentes no jogo obedecem a um padrão desde o início, } \\
\text { mas sem torná-lo previsível demais e desinteressante. }\end{array}$ & $\begin{array}{l}\text { [Valle et. al, 2013] } \\
\text { [Souza et. al, 2010] }\end{array}$ \\
\hline H04- Os elementos do jogo motivam intrinsecamente (autonomia, domínio e & [Valle et. al, 2013] \\
\hline
\end{tabular}


VI Congresso Brasileiro de Informática na Educação (CBIE 2017)

Anais do XXIII Workshop de Informática na Escola (WIE 2017)

\begin{tabular}{|c|c|}
\hline $\begin{array}{l}\text { significado) e extrinsecamente (recompensas) o jogador à reconhecer, } \\
\text { diagnosticar e se recuperar dos erros cometidos. }\end{array}$ & [Souza et. al, 2010] \\
\hline HEURÍSTICA CRIADAS - HAJED-TDAH & Referência \\
\hline $\begin{array}{l}\text { H05- O jogo estimula a concentração, o raciocínio lógico, o desenvolvimento de } \\
\text { estratégias e a persistência, em níveis crescentes de dificuldade para o jogador. }\end{array}$ & $\begin{array}{c}\text { [Alves e Bonfim, 2016] } \\
\text { [Guimarães e Ribeiro, 2010] }\end{array}$ \\
\hline $\begin{array}{l}\text { H06- O efeito sonoro é condizente com o ambiente do jogo e adequado para } \\
\text { manter a concentração e atenção do jogador. }\end{array}$ & $\begin{array}{c}\text { [Souza et. al, 2010] } \\
\text { [Guimarães e Ribeiro, 2010] }\end{array}$ \\
\hline $\begin{array}{l}\text { H07-O cenário é bem elaborado, sem sobrecarga de elementos que podem } \\
\text { desviar a atenção do jogador e que o deixe perdido, quanto aos objetivos do } \\
\text { jogo. }\end{array}$ & $\begin{array}{c}\text { [Souza et. al, 2010] } \\
\text { [Guimarães e Ribeiro, 2010] }\end{array}$ \\
\hline $\begin{array}{l}\text { H08-O cenário apresenta cores com intensidade forte, que despertam a atenção, } \\
\text { como também ajudam a guiar as ações do jogador para o cumprimento dos } \\
\text { objetivos do jogo. }\end{array}$ & $\begin{array}{l}\text { [Oliveira, Ishitani e Cardoso, } \\
\text { 2013] } \\
\text { [Guimarães e Ribeiro, 2010] }\end{array}$ \\
\hline $\begin{array}{l}\text { H15- O jogo estimula um raciocínio de organização, por meio da identificação, } \\
\text { classificação, seleção e agrupamento de elementos. }\end{array}$ & $\begin{array}{l}\text { A H15 foi criada a partir das } \\
\text { considerações dos } \\
\text { especialistas. }\end{array}$ \\
\hline $\begin{array}{l}\text { H16- A identidade adquirida pelo jogador promove uma sensação de imersão, } \\
\text { dando sentido as tarefas propostas (com conteúdo educacional implícito), para } \\
\text { que o aprendizado possa ser aplicado em outros contextos. }\end{array}$ & $\begin{array}{l}\text { A H16 foi criada a partir das } \\
\text { considerações dos } \\
\text { especialistas. }\end{array}$ \\
\hline $\begin{array}{l}\text { H17- Os elementos do jogo ajuda a promover uma boa interatividade com o } \\
\text { jogador, considerando a faixa etária do público para qual o jogo foi criado. }\end{array}$ & $\begin{array}{l}\text { A H17 foi criada a partir das } \\
\text { considerações dos } \\
\text { especialistas. }\end{array}$ \\
\hline $\begin{array}{l}\text { H18- O jogo apresenta uma proposta atrativa com encadeamento de estímulos } \\
\text { para trabalhar a capacidade de deliberação, a atenção e a memória operacional } \\
\text { do jogador, aumentando a complexidade ao longo do progresso do mesmo no } \\
\text { jogo. }\end{array}$ & $\begin{array}{l}\text { A H18 foi criada a partir das } \\
\text { considerações dos } \\
\text { especialistas. }\end{array}$ \\
\hline $\begin{array}{l}\text { H19- O jogo demonstra o desempenho alcançado pelo usuário, por meio de uma } \\
\text { interface clara e compreensível }\end{array}$ & $\begin{array}{c}\text { A H19 foi criada a partir das } \\
\text { considerações dos } \\
\text { especialistas. }\end{array}$ \\
\hline $\begin{array}{l}\text { H20- As instruções apresentadas no iníci } \\
\text { visualização, fácil acesso e linguagem objet }\end{array}$ & $\begin{array}{c}\text { A H20 foi criada a partir das } \\
\text { considerações dos } \\
\text { especialistas. }\end{array}$ \\
\hline
\end{tabular}

Tabela 1 (CONT.). Conjunto de heurísticas HAJED-TDAH

\begin{tabular}{|l|c|}
\hline \multicolumn{1}{|c|}{ HEURÍSTICA ADAPTADAS - HAJED-TDAH } & Referência \\
\hline $\begin{array}{l}\text { H09-O jogo permite ao usuário reconhecer de maneira atrativa quais são os } \\
\text { objetivos de aprendizagem ou treino comportamental contemplados pelos seus } \\
\text { elementos. }\end{array}$ & [Valle et. al, 2013] \\
\hline $\begin{array}{l}\text { H10-O jogo permiti que seus usuários possam ter maior autonomia do processo } \\
\text { de aprendizagem, (re)configurando suas atividades, objetivos de aprendizagem, } \\
\text { entre outros, desde que oriente de forma explicativa sobre as escolhas tomadas } \\
\text { pelos usuários. }\end{array}$ & [Valle et. al, 2013] \\
\hline $\begin{array}{l}\text { H11-Os elementos do jogo são criados abordando estratégias para aperfeiçoar as } \\
\text { funções executivas, de forma a contemplar diferentes níveis de aprendizagem, } \\
\text { por exemplo, iniciante, intermediário e avançado. }\end{array}$ & [Valle et. al, 2013] \\
\hline $\begin{array}{l}\text { H12-O jogo possui elementos que permitam ao usuário reconhecer seu } \\
\text { progresso, incentivando a persistência ao longo do processo de aprendizagem. }\end{array}$ & [Valle et. al, 2013] \\
\hline $\begin{array}{l}\text { H13-O jogo oferece ao usuário a possibilidade de escolher o nível de } \\
\text { dificuldade do mesmo, explicando de maneira atrativa as dificuldades de cada }\end{array}$ & [Valle et. al, 2013] \\
\hline
\end{tabular}


VI Congresso Brasileiro de Informática na Educação (CBIE 2017)

Anais do XXIII Workshop de Informática na Escola (WIE 2017)

um para evitar frustração.

H14- O jogo possui característica de adaptabilidade, no sentido de que os pontos fortes e fracos dos usuários com TDAH são reconhecidos e os elementos do jogo se adaptam a eles, permitindo ao mesmo autoconhecimento durante a partida.

Conforme pode ser observado na Tabela 1, as heurísticas classificadas como combinadas, tiveram como base as heurísticas HEDEG propostas por Valle et al. (2013) e as recomendações heurísticas destinadas para auxiliar na avaliação ou adequação de jogos às capacidades cognitivas de PNEEs (Pessoas com Necessidades Educacionais Especiais), criadas por Souza et al. (2010).

As heurísticas que foram criadas, por meio deste estudo, tiveram como base: i) a análise dos trabalhos de Guimarães e Ribeiro (2010), Alves e Bonfim (2016) e Oliveira, Ishitani e Cardoso (2013), que descrevem sobre quais aspectos devem ser considerados na criação desses jogos para atender as especificidades deste público; ii) características elencadas junto aos especialistas.

Dentre as características, relatadas pelos especialistas, pode-se destacar que pessoas com TDAH possuem dificuldades em relação a organização e que esse comportamento pode ser melhorado "treinando o cérebro" de várias maneiras, envolvendo a identificação, classificação, seleção e agrupamento de componentes presentes no jogo.

Outra característica, ressaltada pelos especialistas, é a dificuldade que as pessoas com TDAH possuem ao se depararem com situações que exijam análise de prós e contras. Sendo assim, um jogo que estimula habilidades como: deliberação, atenção e memória operacional pode ser capaz de simular um treino comportamental no jogador, por meio de estímulos e respostas que aumentam gradativamente de dificuldade.

\section{Avaliação do conjunto de heurísticas HAJED-TDAH}

Nesta seção são apresentados a definição, condução e análise dos resultados da avaliação do conjunto de heurísticas HAJED-TDAH

\subsection{Definição da Avaliação}

Para a realização dessa avaliação, foram elaboradas duas hipóteses, conforme pode ser observado na Tabela 2.

Tabela 2. Hipóteses do Estudo Avaliativo

\begin{tabular}{|c|c|}
\hline $\begin{array}{l}\text { H0 } \\
\text { (HIPÓTESE NULA) } \\
\text { Não há diferença }\end{array}$ & $\begin{array}{l}\text { Entre se utilizar ou não o conjunto de heurísticas HAJED-TDAH por não } \\
\text { especialistas, com relação à eficiência para identificação de problemas, ou seja } \\
\text { EHAJED-TDAH=ELIVRE }\end{array}$ \\
\hline $\mathrm{H} 1$ & \\
\hline $\begin{array}{c}\text { (HIPÓTESE } \\
\text { ALTERNATIVA) }\end{array}$ & $\begin{array}{l}\text { Em utilizar o conjunto de heurísticas HAJED-TDAH por não especialistas, com } \\
\text { relação à eficiência para identificação de problemas, ou seja EHAJED-TDAH\#ELIVRE }\end{array}$ \\
\hline Há diferença & \\
\hline
\end{tabular}


No contexto desta avaliação, eficiência está relacionada a quão próximo está o grau de severidade atribuído pelos usuários não especialistas do grau de severidade atribuído pelos usuários especialistas. Isso é importante, pois como a avaliação também foi conduzida por não especialistas, pode ser que eles tenham identificado problemas com graus de severidade maior ou menor do que devia ser.

Para a condução, desta avaliação, foram utilizados três grupos de três de avaliadores cada um. Um grupo foi composto por três especialistas em TDAH, e outros dois grupos A e B, foram compostos por não especialistas. Para avaliação de cada grupo, foram utilizados alguns documentos, como: o formulário de caracterização de perfil, para verificar o conhecimentos dos avaliadores sobre questões relacionadas aos assuntos "Avaliação Heurísticas", "Jogos Educacionais Digitais" e "TDAH"; o formulário para anotações, utilizados pelos especialistas anotarem o grau de severidade de cada problema encontrado no jogo; o roteiro do jogo, que apresenta uma lista de atividades/ações para conduzir os avaliadores enquanto avaliam o jogo; e por fim o formulário de avaliação, nesse documento é apresentado o conjunto de heurísticas HAJED-TDAH e os graus de severidade que deve-se atribuir a cada problema representado por cada heurística.

\subsection{Condução da Avaliação}

A primeira etapa da avaliação consistiu na avaliação do jogo pelos três especialistas em TDAH. Cada avaliador especialista preencheu um formulário de anotações para atribuir um grau de severidade a cada problema encontrado, e o roteiro do jogo Guardiões da Floresta. Conforme a avaliação dos especialistas, foram encontrados 15 problemas e a soma do grau de severidade foi 29.

Na segunda etapa da avaliação, os avaliadores não especialistas foram divididos em dois grupos, em que um grupo composto por duas alunas do curso de Psicologia com experiência em TDAH e uma aluna do curso de Ciências da Computação com experiência em jogos digitais (Grupo A). As integrantes avaliaram o jogo assim como os especialistas, utilizando somente o roteiro do jogo e um formulário para anotações dos problemas encontrados e dos seus respectivos graus de severidade. Conforme avaliação deste grupo, foram identificados 6 problemas e a soma do grau de severidade foi 17 .

A última etapa, deste estudo avaliativo, foi realizada por avaliadores não especialistas composto por três alunos do curso de Ciências da Computação com experiência somente em jogos digitais (Grupo B), mas que realizaram um treinamento sobre as principais características do TDAH, eles utilizaram o conjunto de heurísticas HAJED-TDAH e o roteiro do jogo. Como resultado final, deste grupo, foram identificados 10 problemas com a soma do grau de severidade igual a 15 .

\subsection{Análise dos resultados}

Observando as avaliações dos grupos, constatou-se que os integrantes do Grupo B conseguiram encontrar uma quantidade maior de problemas do que os integrantes Grupo A, que realizaram de forma livre a avaliação do jogo, ou seja, sem o auxílio do conjunto HAJED-TDAH.

Para possibilitar uma comparação final dos Grupos (A e B) e comprovar a eficiência do Conjunto HAJED-TDAH, foi utilizado como parâmetro o Valor 
VI Congresso Brasileiro de Informática na Educação (CBIE 2017)

Anais do XXIII Workshop de Informática na Escola (WIE 2017)

Relacional - VR (representado pela Fórmula 1) entre o valor do somatório dos Graus de Severidade (GS) com a quantidade de problemas encontrados (QtP), respectivos de cada grupo.

$$
\text { (1) } \mathrm{VR}=\sum \mathrm{GS} / \mathrm{QtP}
$$

Desta forma, a comparação dos resultados obtidos pelos três grupos é apresentada na Tabela 3.

Tabela 3. Comparação dos resultados dos grupos A, B e especialistas

\begin{tabular}{|l|l|l|l|l|}
\hline \multicolumn{1}{|c|}{ Grupo } & \multicolumn{1}{c|}{ Qtd. Problemas } & Soma Graus Severidade & \multicolumn{1}{c|}{ VR } \\
\hline Especialistas & 15 & 29 & 1,93 \\
\hline Grupo A & 6 & 17 & 2,83 \\
\hline Grupo B & 10 & 15 & 1,5 \\
\hline
\end{tabular}

Portanto, como pode ser visto na Tabela 3, o resultado do Grupo B alcançou um valor mais próximo do VR resultante da avaliação dos especialistas, confirmando a hipótese (H1) levantada neste trabalho. No entanto, foi necessário verificar se os problemas encontrados pelo grupo que utilizou o conjunto de heurísticas HAJEDTDAH foram semelhantes aos problemas encontrados pelos especialistas, que representa o oráculo desta pesquisa. Foi possível perceber que algumas das heurísticas do conjunto HAJED-TDAH, mais especificamente dez heurísticas, coincidiram com os problemas relatados pelos especialistas.

A Tabela 4 apresenta quais heurísticas do conjunto HAJED-TDAH abordam os mesmos problemas apontados pelos especialistas, sendo que algumas delas se repetem para problemas distintos, como é o caso das heurísticas H01, H11, H16 que representam os problemas $(1 ; 10 ; 11),(12 ; 13)$, e $(4 ; 6)$ respectivamente.

Tabela 4. Comparação das heurísticas HAJED-TDAH e observações especialistas

\begin{tabular}{|c|c|c|}
\hline $\begin{array}{c}\text { HAJED } \\
\text {-TDAH }\end{array}$ & Comentários Especialistas \\
\hline H01 & $\begin{array}{c}\text { Essa falha na visualização dos diálogos dos personagens compromete o entendimento do jogador, } \\
\text { como também perde a utilidade de transmitir informaços do jogo para conduzi-lo durante a partida. }\end{array}$ \\
\hline H19 & $\begin{array}{c}\text { Este problema está relacionada ao feedback do jogo, critério essencial para que o jogador entenda sua } \\
\text { atuação durante a partida. Segundo as características de Annetta (2010), a análise de desempenho, } \\
\text { deve ser exposta para o jogador de modo que ele consiga entender o retorno dado pelo jogo. }\end{array}$ \\
\hline H12 & $\begin{array}{c}\text { Essa heurística também trata o problema apontado pelos especialistas sobre atratividade do jogo, e } \\
\text { também como o jogador é incentivado a permanecer jogando, principalmente as pessoas que possuem } \\
\text { TDAH, que são mais suscetíveis a abandonarem uma tarefa quando não são atraídas pela mesma. }\end{array}$ \\
\hline H16 & $\begin{array}{c}\text { A heurística apresenta a mesma questão levantada como problema pelos especialistas, mas também } \\
\text { reflete sobre a influência da identidade adquirida pelo jogador enquanto joga, se ela provoca um } \\
\text { imersão tal, que possibilite ao jogador perceber quais dificuldades estão sendo trabalhadas, para que } \\
\text { essa melhora possa atingir outros contextos, como por exemplo a sala de aula. }\end{array}$ \\
\hline H16 & $\begin{array}{c}\text { A heurística do conjunto HAJED-TDAH relacionada ao problema de imersão é a "H16", no caso a } \\
\text { crítica realizada pelos especialistas foi em relação a parte book do Gamebook e não a parte do jogo e } \\
\text { nem dos minigames, mesmo assim a consideração é relevante, já que é justamente nessa parte que o } \\
\text { jogador pode se entediar e abandonar o jogo. }\end{array}$ \\
\hline H09 & $\begin{array}{c}\text { Essa heurística corresponde ao problema apontado pelos especialistas a respeito do intertexto entre o } \\
\text { conteúdo da história e o jogo, em que devem estar correlacionados a atratividade dos elementos, para }\end{array}$ \\
\hline
\end{tabular}


VI Congresso Brasileiro de Informática na Educação (CBIE 2017)

Anais do XXIII Workshop de Informática na Escola (WIE 2017)

\begin{tabular}{|c|c|c|}
\hline H20 & que os objetivos do jogo sejam alcançados. \\
\hline H01 & $\begin{array}{c}\text { A heurística representa a questão de que pessoas com TDAH precisam de orientação adequada as } \\
\text { dificuldades do transtorno, para que assim eles consigam cumprir os objetivos do jogo sem } \\
\text { frustrações. }\end{array}$ \\
\hline H11 & $\begin{array}{c}\text { A heurística corresponde ao problema sobre os recursos apresentados pelo jogo devem despertar a } \\
\text { atenção do jogador, para que ele não fique perdido ao jogar, e quanto a funcionalidade da agenda, se o } \\
\text { jogador não entender qual sua utilidade, dificilmente ela chamará sua atenção e consequentemente o } \\
\text { recurso será desperdiçado. }\end{array}$ \\
\hline H03 & $\begin{array}{c}\text { A heurística descreve sobre a importância do jogo trabalhar as funções executivas em níveis } \\
\text { crescentes de dificuldade. Segundo Annetta (2010) a complexidade dos desafios do jogo deve ser } \\
\text { apropriada para cada nível e público, e no caso de pessoas com TDAH este fator é mais meticuloso } \\
\text { ainda, pois dependendo da dificuldade do jogo, o jogador não consegue realizar o mínimo requerido, } \\
\text { no entanto pode nem se interessar por jogos que não ofereçam desafios atrativos. }\end{array}$ \\
\hline H17 & $\begin{array}{c}\text { A heurística diz respeito a coerência do jogo com relação ao TDAH. Segundo os especialistas esse } \\
\text { aspecto é muito importante e o jogo não apresentou problema relacionado a esse ponto, mas ele foi } \\
\text { citado para mostrar que o assunto é um requisito a ser avaliado. }\end{array}$ \\
\hline $\begin{array}{c}\text { A heurística do conjunto HAJED-TDAH que representa essa questão é a "H17", que trata sobre faixa } \\
\text { etária que o jogo pretende alcançar. Segundo os especialistas o jogo não apresentou problema } \\
\text { relacionado a faixa etária destinada, porem como na observação anterior, foi citado para manifestar a } \\
\text { importância de avaliar esse critério. }\end{array}$ \\
\hline
\end{tabular}

Conforme foi apresentado, após uma comparação entre às avaliações realizadas pelo grupo de especialistas e o grupo $\mathrm{B}$, foram encontradas dez heurísticas do conjunto HAJED-TDAH que representam treze problemas do jogo apontados pelo especialistas. Dos quinze problemas apontados pelos especialistas, dois não foram contemplados pelas heurísticas do conjunto HAJED-TDAH, sendo eles: o problema 5 "Instruções adequadas para crianças" e 8 "A duração do jogo é um fator importante, crianças com TDAH, não se concentram por muito tempo em uma atividade, especialmente se não as desafiam". O problema 5, segundo os especialistas, diz respeito ao poder de influência da história, considerando que o jogo narra como a personagem principal "Lyu" se perdeu dos pais na Floresta Amazônica e não demonstra nenhum receio ao conversar com estranhos, no caso os personagens guardiões da floresta. Assim, os especialistas consideram importante, observar esses aspectos de influência para crianças que não desenvolveram senso crítico diante de situações como essa, principalmente crianças com TDAH que são mais suscetíveis a não medirem consequências de suas atitudes. Já o problema 8 relacionado a duração do jogo, os especialistas questionaram sobre o jogo apresentar fases longas, o que pode prejudicar a concentração dos jogadores com TDAH. As demais heurísticas do conjunto HAJED-TDAH contemplam aspectos para análise de jogos em geral, provavelmente os especialistas se preocuparam em avaliar problemas específicos do jogo relacionado ao TDAH.

O grupo A, composto por avaliadores não especialistas que não utilizaram das heurísticas do conjunto HAJED-TDAH para avaliar o jogo. Pode se observar que os problemas encontrados por este grupo foram descritos de forma subjetiva, além de apresentar conclusões sérias sobre o jogo, que nem mesmo os especialistas afirmaram, como é o caso do problema 5 "Não tem atração suficiente, ou seja, não atrai uma criança com TDAH" e o problema 3 "Não apresenta um interface intuitiva, visto que é um jogo infantil". Apesar disso, o grupo conseguiu identificar um problema relatado pelos especialistas, sendo este o problema 4 "Não demonstra pontuação final do usuário", mas 
também houve divergência sobre a questão da faixa etária para qual o jogo foi criado, como descreve o problema 2 "Não atinge muitas faixas etárias, apenas crianças com idade inferior a 7 anos", diferentemente da conclusão dos especialistas em TDAH, que consideraram o jogo adequado à faixa etária estabelecida pelos desenvolvedores do Guardiões da Floresta.

\section{Considerações Finais}

As avaliações realizadas em JEDs, desenvolvidos para pessoas com TDAH, devem obedecer a mesma linha de comportamento das avaliações de jogos digitais em geral, sendo necessário que esses JEDs também sejam avaliados de maneira confiável, principalmente para evitar a utilização de jogos de má qualidade pelos educadores e tentar impedir possíveis frustrações dos alunos com TDAH.

Sendo assim, com intuito de amenizar o problema relacionado as avaliações de JEDs para pessoas com TDAH, foi proposto por este trabalho o conjunto de heurísticas HAJED-TDAH, para avaliação e desenvolvimento de JEDs para pessoas com TDAH.

O conjunto de heurísticas HAJED-TDAH foi validado por avaliadores especialistas e não especialistas, sendo aplicado no jogo específico para pessoas com TDAH, denominado Guardiões da Floresta.

Ao analisar os resultados finais do processo avaliativo, foi possível observar que o grupo de avaliadores não especialistas, que fez uso das heurísticas HAJED-TDAH, obteve resultados mais próximos aos resultados alcançados por especialistas, corroborando com a Hipótese (H1), levantada por este estudo, e comprovando a eficiência do conjunto de heurísticas HAJED-TDAH com relação ao seu objetivo de promover uma melhor avaliação de JEDs desenvolvidos para pessoas com TDAH, bem como servir como um guia de desenvolvimento para este gênero de jogo. 
VI Congresso Brasileiro de Informática na Educação (CBIE 2017)

Anais do XXIII Workshop de Informática na Escola (WIE 2017)

\section{Referências}

ALVES, L.; BONFIM, C. e a estimulação de funções executivas em crianças com indicação de diagnóstico de tdah: Processo de pré-produção, produção e avaliação do software. Revista da FAEEBA-Educação e Contemporaneidade, v. 25, n. 46, 2016.

ANNETTA, L. A. The "i's" have it: A framework for serious educational game design. Review of General Psychology, Educational Publishing Foundation, v. 14, n. 2, p. 105, 2010 .

BASTOS, A. P. Z. et al. Utilização de um jogo sério e naïve bayes para auxiliar na avaliação cognitiva do transtorno de déficit de atenção/hiperatividade. In: Anais do Simpósio Brasileiro de Informática na Educação. [S.1.: s.n.], 2012. v. 23, n. 1.

GUIMARÃES, M.; RIBEIRO, P. Utilização de jogos virtuais na prática educacional de crianças com transtorno de déficit de atenção e/ou hiperatividade. [S.1.]: SBGames, 2010.

LEITE, P.; MENDONÇA, V. Diretrizes para game design de jogos educacionais. Proceedings of SBGames, Art \& Design Track, Full Papers, 2013.

NIELSEN, J. Heuristic evaluation. Usability inspection methods, v. 17, n. 1, p. 25-62, 1994.

OLIVEIRA, L. B.; ISHITANI, L.; CARDOSO, A. M. Jogos computacionais e transtorno de déficit de atenção e hiperatividade: Revisão sistemática de literatura. Nuevas Ideas en Informática Educativa TISE, 2013.

RAMOS, D. K. Jogos cognitivos eletrônicos: contribuições à aprendizagem no contexto escolar. Ciências \& Cognição, Instituto de Ciências Cognitivas, v. 18, n. 1, p. 19-32, 2013.

RIVERO, T. S.; QUERINO, E. H. G.; STARLING-ALVES, I. Videogame: seu impacto na atenção, percepção e funções executivas. Neuropsicologia Latinoamericana, v. 4, n. 3, 2012.

ROCHA, P.; FILHO, J. N.; ALVES, L. Jogos digitais e reabilitação neuropsicológica: delineando novas mídias. Anais do Seminário Tecnologias Aplicadas a Educação e Saúde, v. 1, n. 1, 2014.

ROCHA, R. V. da; BITTENCOURT, I. I.; ISOTANI, S. Análise, projeto, desenvolvimento e avaliação de jogos sérios e afins: uma revisão de desafios e oportunidades. In: Anais do Simpósio Brasileiro de Informática na Educação. [S.1.: s.n.], 2015. v. 26, n. 1, p. 692.

SILVA, R. A.; PAULA, M. M.; SARLAS, L. M. Utilização de jogos para pessoas com necessidades educativas especiais: uma análise experimental. In: Anais do Simpósio Brasileiro de Informática na Educação. [S.1.: s.n.], 2011. v. 1, n. 1.

SOUZA, F. F. de et al. Necessidades educativas especiais: construção de jogos para apoiar o aprendizado. In: IX SBGames-Trilha de Games \& Cultura Full Papers. [S.1.: s.n.], 2010. p. 134-142.

VALLE, P. H. D. et al. Hedeg-heurísticas para avaliação de jogos educacionais digitais. Nuevas Ideas en Informática Educativa TISE, 2013. 